\title{
Psychiatric Comorbidity in Patients with Psoriasis, Vitiligo, Acne, Eczema and Group of Patients with Miscellaneous Dermatological Diagnoses
}

\author{
Amir Mufaddel1,2*, Abdelghani Elsheikh Abdelgani ${ }^{3}$ \\ ${ }^{1}$ Community Mental Health Services, Behavioral Sciences Institute, Al Ain Hospital, Al Ain, UAE \\ ${ }^{2}$ United Arab Emirates University, Al Ain, UAE \\ ${ }^{3}$ Department of Psychiatry, Faculty of Medicine, Ribat National University, Khartoum, Sudan \\ Email: ${ }^{*}$ khalifaamir@yahoo.co.uk
}

Received 20 April 2014; revised 18 May 2014; accepted 15 June 2014

Copyright (C) 2014 by authors and Scientific Research Publishing Inc.

This work is licensed under the Creative Commons Attribution International License (CC BY). http://creativecommons.org/licenses/by/4.0/

(c) (i) Open Access

\section{Abstract}

Background: Dermatological conditions can be associated with high psychiatric comorbidity. Several studies reported high rates of depression and anxiety particularly for specific dermatological disorders such as psoriasis and acne. Aim: The aim of this study was to compare the rates of psychiatric symptoms in patients with psoriasis, acne, vitiligo, and eczema versus patients who had other dermatological conditions; and to compare each dermatological group versus healthy control subjects. Methods: This prospective cross-sectional study was conducted in dermatology outpatient clinics in Khartoum. Hospital Anxiety and Depression Scale (HADS) was used to assess symptoms of anxiety (HADS-A) and depression (HADS-D). ICD-10 criteria were used for clinical psychiatric diagnosis. Tabulated results were analyzed using Chi-square test. Significance was set at $P<0.05$. Results: HADS-D scores above the cut off points were significantly higher in patients with psoriasis $(P=0.0062)$, vitiligo $(P=0.0054)$, acne $(P=0.0103)$ and eczema $(P=0.0359)$ compared with healthy subjects. Similarly, HADS-A scores above the cut off points were significantly higher in patients with psoriasis $(P<0.0 .0001)$, vitiligo $(P=0.0001)$, acne $(P=0.0143)$ and eczema $(P=0.0281)$ compared with healthy subjects. No significant difference between the control group and patients with other dermatologic conditions regarding both HADS-D and HADS-A scores. Using ICD-10 criteria for clinical psychiatric diagnoses indicated that $52.3 \%$ of dermatology patients had an associated ICD-10 diagnosis; most commonly anxiety disorders (28.6\%), and depression (21.9\%). ICD-10 diagnoses of anxiety disorders included: OCD (13.3\%) generalized anxiety disorder $(5.7 \%)$, panic disorder $(4.8 \%)$, phobic anxiety disorder $(3.8 \%)$ and post-traumatic stress disorder $(0.95 \%)$. Conclusion: Dermatological conditions are associated with high rates of

${ }^{*}$ Corresponding author.

How to cite this paper: Mufaddel, A. and Abdelghani, A.E. (2014) Psychiatric Comorbidity in Patients with Psoriasis, Vitiligo, Acne, Eczema and Group of Patients with Miscellaneous Dermatological Diagnoses. Open Journal of Psychiatry, 4, 168-175. http://dx.doi.org/10.4236/ojpsych.2014.43022 
psychiatric comorbidity. Screening for anxiety and depressive symptoms may be helpful for early diagnosis and management of associated psychiatric symptoms.

\title{
Keywords
}

\author{
Psychiatric Comorbidity, Dermatologic Disorders, Anxiety, Depression
}

\section{Background}

Several dermatological conditions have been reported in association with psychological stress such as psoriasis, alopecia areata, atopic dermatitis, lichen planus, pruritus and rosacea. Moreover, emotional stress may exacerbate many chronic dermatologic conditions and can also lead to initiation of a vicious cycle referred to as "itchscratch cycle" [1] [2]. Psychiatric and psychological factors are thought to play a role in at least $30 \%$ of dermatologic disorders [3].

It is well-known that psychological stress leads to activation of the HPA axis which can result in undesirable physiological responses including the exacerbation of dermatological conditions. HPA axis and the sympathetic nervous system can modulate the cutaneous immune responses, and the psychological stress can affect development and progression of skin diseases [4]. The mechanism by which stress affects the inflammatory process in the skin is not well understood. The activity of mast cells, Natural killer (NK) cells or dendrites in the skin are influenced by neuroendocrine mediators including Corticotrophin Releasing Factor (CRF), adrenocorticotropic hormone (ACTH), substance P, glucocorticoids and catecholamines [5].

Cortisol and norepinephrine release have influence on the immune system through regulating $\mathrm{T}$ cells and macrophages differentiation and through modifying inflammation and immunity. Reduced HPA-axis reactivity could be a general phenomenon associated with all allergic conditions and not specific to the skin as in the case of allergic asthma and allergic dermatitis which have parallel immunopathology and blunted cortisol responses [6].

In some of the dermatological disorders, such as acne and psoriasis, the psychiatric co-morbidity is an important measure of the overall patient's disability; and they can be associated with psychiatric emergencies including risk of suicide [3].

Patients with dermatological diseases are more likely to report anxiety, depressive symptoms and suicidal ideation than those without chronic dermatological conditions. For example, one study documented that the prevalence of active suicidal ideation among psoriasis (7.2\%) and acne (5.6\%) patients was higher than patients with general medical conditions (2.4\% - 3.3\%) [7]. Patients with psoriasis are frequently distressed by symptoms of itching and scratching, bleeding, physical appearance and flakes which have substantial effects on their lives [8].

The prevalence of depression is 2 to 3 times greater in acne patients than in the general population, which is more common in patients older than 36 and female patients [9].

Additionally, Isotretinoin which is used for treatment of acne is thought to be associated with increased risk for depression and suicidal attempts [10].

\section{Methods}

The current study is a cross-sectional observational study conducted in Khartoum Teaching Hospital, dermatology department. The aim of the study was to compare the levels of anxiety and depressive symptoms in patients with psoriasis, acne, vitiligo, and eczema with those who had other dermatological conditions; and to compare each dermatological group with a control group of healthy subjects. The study was approved by the Sudan Medical Specialization Board (SMSB)/council of psychiatry. The nature of the study and its objectives were explained to participants and an informed consent was signed by all participants before being recruited in the study.

\subsection{Participants}

We recruited 105 consecutive patients with dermatological conditions and 105 control subjects with no current 
history of dermatological disease. The dermatological diagnosis was done by the dermatologist in the clinic and, based on the dermatological diagnosis, the patients were divided into 5 groups including psoriasis, acne, vitiligo and eczema; in addition to a fifth group composed of patients with other dermatological conditions. The patients' group included 51 (48.6\%) males and 54 (51.4\%) females. The majority (66 patients/62.8\%) of them aged between 20 and 34 years, and most of them were single (69 patients/ 65.7\%). The healthy control subjects were 53 (51\%) males and 52 (49\%) females. The majority of them (51 subjects/48.6\%) aged between 20 and 34 years; and the majority were single (71 subjects/67.6\%).

\subsection{Instruments}

We used Hospital Anxiety \& Depression Scale (HADS) as psychometric instrument to compare levels of depression and anxiety in the 5 groups of patients and the healthy control group. HADS is a self-report questionnaire which was developed by Zigmond et al., in 1983 and is commonly used to assess levels of anxiety and depression [11]. HADS questionnaire consists of 14 questions; 7 questions assessing the level of anxiety (HADS-A) and 7 questions assessing depression (HADS-D). Each question has four options, so patients were asked to choose one response from the four given for each question. The answers for the anxiety were added to give the total score for anxiety, and those for depression were added to give the total score for depression.

A cut-off score of 8+ was used for caseness for both anxiety and depression. The patients were then referred to a psychiatric evaluation including history and mental state examination; ICD-10 criteria were used to make the psychiatric diagnosis.

Tabulated results were analyzed using Chi-square test and Pearson's correlation, $P<0.05$ was considered significant.

\section{Results}

The sample included 19 patients presented with psoriasis, 24 patients with vitilligo, 16 patients with eczema and 20 patients with acne in addition to a fifth group composed of 26 patients with miscellaneous dermatological diagnoses such as cutaneous lishmaniasis, drug eruptions, pemphigus, mycosis fungoid, urticaria, alopecia areata, ...etc.

Table 1 compares the scores for depression in patients presenting with different dermatological conditions versus healthy control subjects. HADS-D scores with cut off points of $8+$ were found significantly higher in patients with psoriasis $(P=0.0062)$, vitiligo $(P=0.0054)$, acne $(P=0.0103)$ and eczema $(P=0.0359)$ compared with healthy subjects.

Table 2 compares the anxiety scores in patients presenting with different dermatological conditions versus healthy control subjects. HADS-A scores with cut off points of $8+$ were also found significantly higher in patients with psoriasis $(P<0.0 .0001)$, vitiligo $(P=0.0001)$, acne $(P=0.0143)$ and eczema $(P=0.0281)$ compared with healthy subjects.

No significant differences were found between the control group and patients with other dermatologic conditions (miscellaneous group) regarding both HADS-D and HADS-A scores.

Psychiatric history and mental state examination for all patients indicated that about $52.3 \%$ of patients with dermatological diseases had an associated ICD-10 diagnosable psychiatric morbidity. The most commonly frequent ICD-10 diagnoses were anxiety disorders which occurred in (28.5\%), and depression (21.9\%). The overall

Table 1. HADS depression scores in patients with different dermatological conditions compared with control subjects.

\begin{tabular}{lccc}
\hline & $\begin{array}{c}\text { HADS-D } \\
\text { Scores 8+ }\end{array}$ & $\begin{array}{c}\text { HADS-D } \\
\text { Scores < 8 }\end{array}$ & $\begin{array}{c}\text { P value } \\
\text { (Dermatological groups Vs Healthy Controls) }\end{array}$ \\
\hline Psoriasis $(\mathrm{n}=19)$ & 09 & 10 & $P=0.0062$ \\
Vitiligo $(\mathrm{n}=24)$ & 12 & 12 & $P=0.0054$ \\
Eczema $(\mathrm{n}=16)$ & 09 & 07 & $P=0.0359$ \\
Acne $(\mathrm{n}=20)$ & 10 & 10 & $P=0.0103$ \\
Other dermatological conditions $(\mathrm{n}=26)$ & 18 & 08 & $P=0.3574$ \\
Control (n=105) & 84 & 21 & \\
\hline
\end{tabular}


Table 2. HADS-anxiety scores in patients with different dermatological conditions compared with control subjects.

\begin{tabular}{cccc}
\hline & $\begin{array}{c}\text { HADS-A } \\
\text { scores }<\mathbf{8}\end{array}$ & $\begin{array}{c}\text { HADS-A } \\
\text { scores 8+ }\end{array}$ & $\begin{array}{c}\text { P value } \\
\text { (Dermatological groups Vs Healthy Control) }\end{array}$ \\
\hline Psoriasis $(\mathrm{n}=19)$ & 05 & 14 & $P<0.0001$ \\
Vitiligo $(\mathrm{n}=24)$ & 09 & 15 & $P=0.0001$ \\
Eczema $(\mathrm{n}=16)$ & 08 & 08 & $P=0.0281$ \\
Acne $(\mathrm{n}=20)$ & 10 & 10 & $P=0.0143$ \\
Miscellaneous $(\mathrm{n}=26)$ & 16 & 10 & $P=0.1084$ \\
Control $(\mathrm{n}=105)$ & 83 & 22 & \\
\hline
\end{tabular}

rate of ICD-10 psychiatric diagnoses was significantly higher in patients with psoriasis $(P=0.0032)$ compared with the miscellaneous group of dermatological conditions (Table 3).

ICD-10 diagnosis of depression was most commonly reported in patients with psoriasis (36.8\%) followed by eczema (31.2\%) and acne (30\%). Significantly higher rates of depression were found among psoriasis group of patients compared with the miscellaneous group of patients $(P=0.0437)$. No statistically significant differences were found between, eczema, acne and vitiligo compared with the miscellaneous group of dermatological disorders (Figure 1).

ICD-10 diagnoses of anxiety disorders were most commonly reported in patients with vitiligo (45.8\%) and psoriasis (42.1\%). Significantly higher rates of anxiety disorders were found in vitiligo group of patients compared with the miscellaneous group of patients $(P=0.0439)$. No statistically significant differences were found between psosriasis, eczema and acne compared with the miscellaneous group of dermatological disorders (Figure 2).

Types of anxiety disorders (Table 4) included: OCD (13.3\%), generalized anxiety disorder (5.7\%), panic disorder (4.8\%), phobic anxiety disorder (3.8\%) and post-traumatic stress disorder (0.95\%).

Other psychiatric diagnoses included one patient of cutaneous lieshmaniasis with past history and current features of schizophrenia, and 14 years old child with vitiligo who had nocturnal enuresis, both accounting for $1.9 \%$ of the total 105 patients.

\section{Discussion}

This is a cross-sectional observational study conducted in dermatological population and its results may not be generalized to other populations. Our findings suggest that there is a high psychiatric comorbidity in patients with a wide range of dermatological conditions with psoriasis being the most dermatological condition associated with psychiatric comorbidity. There are variations in the prevalence of psychiatric disorders in dermatology patients across several studies using different methods and instruments. For example, one study using HADS has estimated the prevalence of psychological symptoms in dermatological patients to be ranging between $25.9 \%$ and 31\% [12], while the prevalence of psychiatric disorders in patients attending a dermatology outpatient clinic, using the 12 -item General Health Questionnaire (GHQ), was found to be $33.4 \%$ in another study [13]. Other two studies using the same instrument (12-item GHQ) reported less but significant psychiatric comorbity in dermatological patients with an overall prevalence of psychiatric comorbidity ranging between 7.6\% and 25.2\% [14] [15]. Higher prevalence estimates were also documented by using GHQ-28 with psychiatric comorbidity approaching 51.3\% [16]. Hughes and colleagues found higher scores of GHQ (60\%) in dermatological inpatients and less scores (30\%) in dermatological outpatients [17].

Using DSM-IV criteria has shown that about 38\% of dermatological patients received a DSM-IV diagnosis; most commonly mood disorders (20\%) and anxiety disorders (16\%). By using Diagnostic Criteria for Psychosomatic Research (DCPR), 48\% of patients have also received a DCPR diagnosis; most commonly demoralization, irritable mood, type A behavior and abnormal illness behavior [18].

Our findings are different in that diagnosis of anxiety disorders in our patients was more frequent than diagnosis of depression. Findings similar to our results were reported by a study using HADS scores indicating higher prevalence of anxiety (28\%) than depression (20\%) [19]. Depressive disorders are common among patients with dermatological disorders occurring in $30 \%$ of cases, which is more frequent compared to patients in general population where the prevalence of depression is about $22 \%$ [20]. 
Table 3. ICD-10 psychiatric diagnoses in patients with psoriasis, vitiligo, eczema and acne compared with patients of other dermatological diagnoses.

\begin{tabular}{lcccc}
\hline & $\begin{array}{c}\text { Received ICD-10 } \\
\text { Psychiatric diagnosis }\end{array}$ & $\begin{array}{c}\text { No psychiatric } \\
\text { diagnosis }\end{array}$ & Total & P value \\
\hline Psoriasis & $15(78.9 \%)$ & 04 & 19 & $P=0.0032$ \\
Vitiligo & $14(58.3 \%)$ & 10 & 24 & $P=0.0927$ \\
Eczema & $09(56.2 \%)$ & 07 & 16 & $P=0.1689$ \\
Acne & $08(40 \%)$ & 12 & 20 & $P=0.7076$ \\
Other dermatological conditions & $09(34.6 \%)$ & 17 & 26 & 105 \\
Total & $55(52.3 \%)$ & 50 & & \\
\hline
\end{tabular}

Table 4. Types of psychiatric diagnoses in patients presented with dermatological conditions.

\begin{tabular}{lcc}
\hline & $\mathbf{N}$ & \% \\
\hline Depression & $\mathbf{2 3}$ & $\mathbf{2 1 . 9 \%}$ \\
Anxiety Disorders: & & $13.3 \%$ \\
$\quad$ OCD & 14 & $05.7 \%$ \\
$\quad$ GAD & 06 & $03.8 \%$ \\
$\quad$ Phobic anxiety disorder & 04 & $04.8 \%$ \\
$\quad$ Panic disorder & 05 & $0.95 \%$ \\
$\quad$ PTSD & 01 & $\mathbf{2 8 . 6 \%}$ \\
Total & $\mathbf{3 0}$ & $\mathbf{0 1 . 9 \%}$ \\
Other psychiatric diagnoses: & $\mathbf{0 2}$ & $\mathbf{4 7 . 6 \%}$ \\
No current psychiatric diagnosis & $\mathbf{5 0}$ & $\mathbf{1 0 0 \%}$ \\
Total & $\mathbf{1 0 5}$ & \\
\hline
\end{tabular}

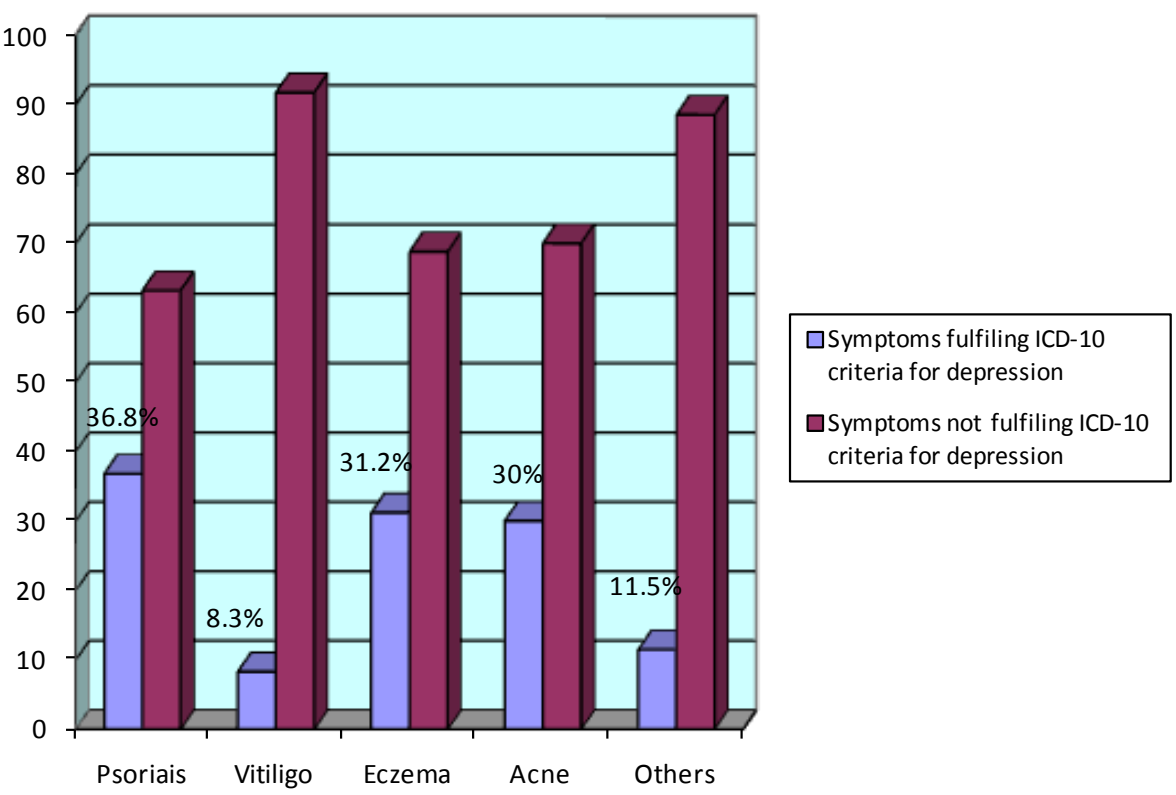

Figure 1. ICD-10 diagnosis of depression in patients with psoriasis, vitiligo, acne, and eczema compared with patients presented with other dermatological conditions. ICD-10 diagnosis of depression was most commonly reported in patients with psoriasis $(36.8 \%)$ followed by eczema (31.2\%) and acne (30\%). Significantly higher rates of depression were found among psoriasis group of patients compared with the miscellaneous group of patients $(P=0.0437)$. No statistically significant differences were found between, eczema $(P=0.1141)$, acne $(P=$ $0.1177)$ and vitiligo $(P=0.7059)$ compared with the miscellaneous group of dermatological disorders. 


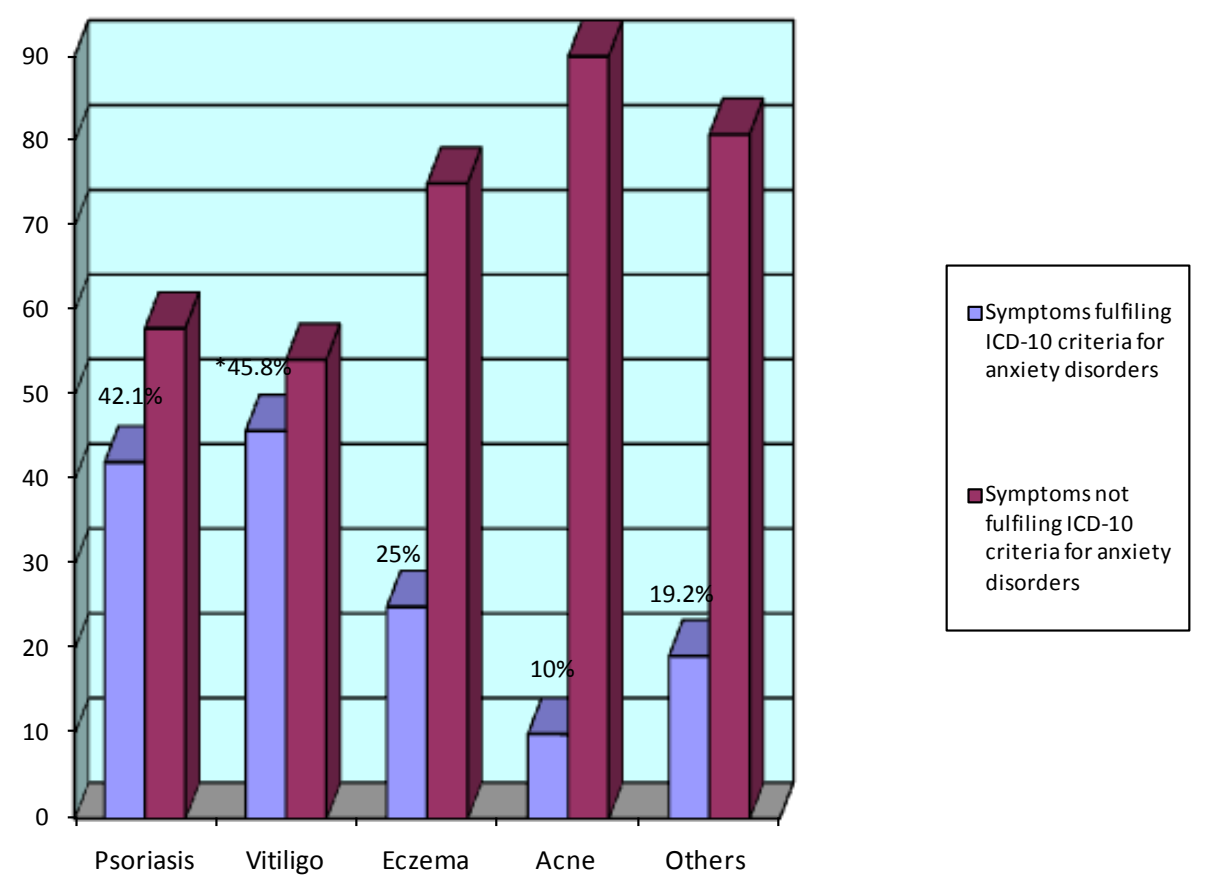

Figure 2. ICD-10 diagnosis of anxiety disorder in patients with psoriasis, vitiligo, acne, and eczema compared with patients presented with other dermatological conditions. ICD-10 diagnoses of anxiety disorders were most commonly reported in patients with vitiligo (45.8\%) and psoriasis (42.1\%). Significantly higher rates of anxiety disorders were found in vitiligo group of patients compared with the miscellaneous group of patients $(P=0.0439)$. No statistically significant differences were found between psoriasis $(P=0.0945)$, eczema $(P=0.6581)$ and acne $(P=0.3876)$ compared with the miscellaneous group of dermatological disorders.

In this study, we found that the most common anxiety disorder among dermatological patients is OCD; followed by GAD, panic attacks/disorder and phobic anxiety disorder. OCD, in our study, was particularly reported in patients with psoriasis, vitiligo and eczema. Itching and self inflicted lesions such as that of neurotic excoriations resemble OCD if it is ritualistic, repetitive, tension reducing and ego-dystonic [21].

Neuroimaging studies in OCD patients have shown activation in cortical and subcortical regions of the orbitofrontal and anterior cingulate loops [22]. The orbitofrontal cortex, supplementary motor and posterior parietal regions were also found to be activated in dermatological patients with disorders characterized by itch and scratch as recently reported by Oxford University researchers who studied the brain areas connected with itch-scratch cycle using functional magnetic resonance imaging (fMRI). Therefore, there are possible similarities in this area of the brain and other disorders that display compulsive behavior such as that occurring in patients with eczema who scratch to the point of harm because they are compelled to do that [23].

Studies suggest the role of interleukins neuropeptides as an associated factor for both stress and dermatologic disorders. For example, Turka and colleagues have demonstrated the presence of interlukin-12 (IL-12) in the skin as well as in the central \& peripheral nervous systems, suggesting that IL-12 can be a mediator to cutaneous reactions to stress [24].

Our findings suggest that ICD-10 diagnosis of depression is more frequently associated with psoriasis, eczema and acne compared with other dermatological diagnoses. This finding supports other studies which documented that depression is known to be associated with psoriasis. One study, using ICD-10 criteria for diagnosis of depression, has shown that depression was diagnosed in $16.9 \%$ of patients with psoriasis vulgaris and was thought to be a reactive depression in the majority of cases [25]. Presence of anxiety and depression in acne patients has been reported by case controlled studies, in which the rates of anxiety (26.2\%) and depression (29.5\%) were significantly higher in acne patients than in the control subjects [26]. Some studies have shown that the highest rates of depressive symptoms and suicidal ideations commonly observed in patients with severe forms of acne and severe psoriasis compared with other dermatological conditions [27]. Mental health problems in acne patients may be related to hormones (androgens) and the fact that acne affects adolescents and people in early 
adult life at the time when they are more likely to be highly concerned with their body images and social lives [28].

\section{Conclusion}

Dermatological disorders are associated with high prevalence of psychiatric comorbidity. Depression and anxiety disorders (such as generalized anxiety, OCD and phobic anxiety disorders) are common in dermatologic patients and they need to be addressed in assessment and management of skin conditions. Screening measures such as HADS questionnaire may be helpful for early diagnosis of anxiety and depressive symptoms but they need to be confirmed by clinical psychiatric evaluation. Early diagnosis of such disabling conditions may have clinical implications enabling clinicians to have collaborative approach to reduce suffering of these patients. Addressing the psychological aspects of dermatological diseases may help minimizing frequency of symptoms exacerbations particularly for disorders known to be exacerbated by stress such as atopic eczema and psoriasis. On the other hand, management of the dermatological symptoms may reduce negative emotional responses, such as anxiety and depression, that can be secondary to the nature of dermatological symptoms.

\section{References}

[1] Jafferany, M. (2007) Psychodermatology: A Guide to Understanding Common Psychocutaneous Disorders. Prim. Care Companion. Journal of Clinical Psychiatry, 9, 203-213.

[2] Osman, O.T., Mufaddel, A., Almugaddam, F. and Augusterfer, E. (2011) The Psychiatric Aspects of Skin Disorders. Expert Review of Dermatology, 6, 195-209. http://dx.doi.org/10.1586/edm.11.10

[3] Gupta, M.A. and Gupta, A.K. (2003) Psychiatric and Psychological Co-Morbidity in Patients with Dermatologic Disorders: Epidemiology and Management. American Journal of Clinical Dermatology, 4, 833-842. http://dx.doi.org/10.2165/00128071-200304120-00003

[4] Hall, J.M., Cruser, D., Podawiltz, A., Mummert, D.I., Jones, H. and Mummert, M.E. (2012) Psychological Stress and the Cutaneous Immune Response: Roles of the HPA Axis and the Sympathetic Nervous System in Atopic Dermatitis and Psoriasis. Dermatology Research and Practice, 2012, Article ID: 403908. http://dx.doi.org/10.1155/2012/403908

[5] Kemeny, M.E. and Scheslowski, M. (2007) Understanding the Interaction between Psychological Stress and Immune-Related Diseases: A Stepwise Progression. Brain, Behavior, and Immunity, 21, 1009-1018. http://dx.doi.org/10.1016/j.bbi.2007.07.010

[6] Buske-Kirschbaum, A. and Hellhammer, D.H. (2003) Endocrine and Immune Responses to Stress in Chronic Inflammatory Skin Disorders. Annals of the New York Academy of Sciences, 992, 231-240. http://dx.doi.org/10.1111/j.1749-6632.2003.tb03153.x

[7] Gupta, M.A. and Gupta, A.K. (1998) Depression and Suicidal Ideation in Dermatology Patients with Acne, Alopecia areata, Atopic Dermatitis and Psoriasis. British Journal of Dermatology, 139, 846-850. http://dx.doi.org/10.1046/j.1365-2133.1998.02511.x

[8] Barankin, B. and DeKoven, J. (2002) Psychological Effect of Common Skin Diseases. Canadian Family Physician, 48, 712-716.

[9] Uhlenhake, E., Yentzer, B.A. and Feldman, S.R. (2010) Acne Vulgaris and Depression: A Retrospective Examination. Journal of Cosmetic Dermatology, 9, 59-63. http://dx.doi.org/10.1111/j.1473-2165.2010.00478.x

[10] Wysowski, D.K., Pitts, M. and Beitz, J. (2001) An Analysis of Reports of Depression and Suicide in Patients Treated with Isotretinoin. Journal of the American Academy of Dermatology, 45, 515-519. http://dx.doi.org/10.1067/mjd.2001.117730

[11] Zigmond, A.S. and Snaith, R.P. (1983) The Hospital Anxiety and Depression Scale. Acta Psychiatrica Scandinavica, 67, 361-370. http://dx.doi.org/10.1111/j.1600-0447.1983.tb09716.x

[12] Windemuth, D., Stücker, M., Hoffmann, K. and Altmeyer, P. (1999) Prevalence of Psychological Symptoms in Dermatologic Patients of an Acute Clinic. Der Hautarzt, 50, 338-343. http://dx.doi.org/10.1007/s001050050917

[13] Aktan, S., Ozmen, E. and Sanli, B. (1998) Psychiatric Disorders in Patients Attending a Dermatology Outpatient Clinic. Dermatology, 197, 230-234. http://dx.doi.org/10.1159/000018002

[14] Picardi, A., Abeni, D., Melchi, C.F., Puddu, P. and Pasquini, P. (2000) Psychiatric Morbidity in Dermatological Outpatients: An Issue to Be Recognized. British Journal of Dermatology, 143, 983-991. http://dx.doi.org/10.1046/j.1365-2133.2000.03831.x

[15] Picardi, A., Abeni, D., Renzi, C., Braga, M., Melchi, C.F. and Pasquini, P. (2003) Treatment Outcome and Incidence of Psychiatric Disorders in Dermatological Out-Patients. Journal of the European Academy of Dermatology and Venere- 
ology, 17, 155-159. http://dx.doi.org/10.1046/j.1468-3083.2003.00619.x

[16] Arbabi, M., Zhand, N., Samadi, Z., Ghaninejad, H. and Golestan, B. (2009) Psychiatric Comorbidity and Quality of Life in Patients with Dermatologic Diseases. Iranian Journal of Psychiatry, 4, 102-106.

[17] Hughes, J.E., Barraclough, B.M., Hamblin, L.G. and White, J.E. (1983) Psychiatric Symptoms in Dermatology Patients. The British Journal of Psychiatry, 143, 51-54. http://dx.doi.org/10.1192/bjp.143.1.51

[18] Picardi, A., Pasquini, P., Abeni, D., Fassone, G., Mazzotti, E. and Fava, G.A. (2005) Psychosomatic Assessment of Skin Diseases in Clinical Practice. Psychotherapy and Psychosomatics, 74, 315-322. http://dx.doi.org/10.1159/000086323

[19] Aslam, R., Qadir, A. and Asad, F. (2007) Psychiatric Morbidity in Dermatological Outpatients: An Issue to Be Recognized. Journal of Pakistan Association of Dermatologists, 17, 235-223.

[20] Filaković, P., Petek, A., Koić, O., Radanović-Grgurić, L. and Degmečić, D. (2009) Comorbidity of Depressive and Dermatologic Disorders-Therapeutic Aspects. Psychiatria Danubina, 21, 401-410.

[21] Gieler, U., Consoli, S.G., Tomas-Aragones, L., Linder, D.M., Jemec, G.B. and Poot, F. (2013) Self-Inflicted Lesions in Dermatology: Terminology and Classification-A Position Paper from the European Society for Dermatology and Psychiatry (ESDaP). Acta Dermato-Venereologica, 93, 4-12.

[22] Rotge, J.Y., Guehl, D., Dilharreguy, B., Cuny, E., Tignol, J., Bioulac, B., Allard, M., Burbaud, P. and Aouizerate, B. (2008) Provocation of Obsessive-Compulsive Symptoms: A Quantitative Voxel-Based Meta-Analysis of Functional Neuroimaging Studies. Journal of Psychiatry and Neuroscience, 33, 405-412.

[23] Leknes, S.G., Bantick, S., Willis, C.M., Wilkinson, J.D., Wise, R.G. and Tracey, I. (2007) Itch and Motivation to Scratch, an Investigation of the Central and Peripheral Correlates of Allergen and Histamine-Induced Itch in Humans. Journal of Neurophysiology, 97, 415-422. http://dx.doi.org/10.1152/jn.00070.2006

[24] Turka, L.A., Goodman, R.E., Rutkowski, J.L., Sima, A.A., Merry, A., Mitra, R.S., Wrone-Smith, T., Toews, G., Strieter, R.M. and Nickoloff, B.J. (1995) Interleukin 12: A Potential Link between Nerve Cells and the Immune Response in Inflammatory Disorders. Molecular Medicine, 1, 690-699.

[25] Pacan, P., Szepietowski, J.C. and Kiejna, A. (2003) Stressful Life Events and Depression in Patients Suffering from Psoriasis Vulgaris. Dermatology and Psychosomatics, 4, 142-145. http://dx.doi.org/10.1159/000073990

[26] Yazici, K., Baz, K. and Yazici, A.E. (2004) Disease-Specific Quality of Life Is Associated with Anxiety and Depression in Patients with Acne. Journal of the European Academy of Dermatology and Venereology, 18, 435-439. http://dx.doi.org/10.1111/j.1468-3083.2004.00946.x

[27] Gupta, M.A. and Gupta, A.K. (1996) Psychodermatology: An Update. Journal of the American Academy of Dermatology, 34, 1030-1046. http://dx.doi.org/10.1016/S0190-9622(96)90284-4

[28] Ritvo, E., Del Rosso, J.Q., Stillman, M.A. and La Riche, C. (2011) Psychosocial Judgments and Perceptions of Adolescents with Acne Vulgaris: A Blinded, Controlled Comparison of Adult and Peer Evaluations. BioPsychoSocial Medicine, 5, 11. http://dx.doi.org/10.1186/1751-0759-5-11 
Scientific Research Publishing (SCIRP) is one of the largest Open Access journal publishers. It is currently publishing more than 200 open access, online, peer-reviewed journals covering a wide range of academic disciplines. SCIRP serves the worldwide academic communities and contributes to the progress and application of science with its publication.

Other selected journals from SCIRP are listed as below. Submit your manuscript to us via either submit@scirp.org or Online Submission Portal.
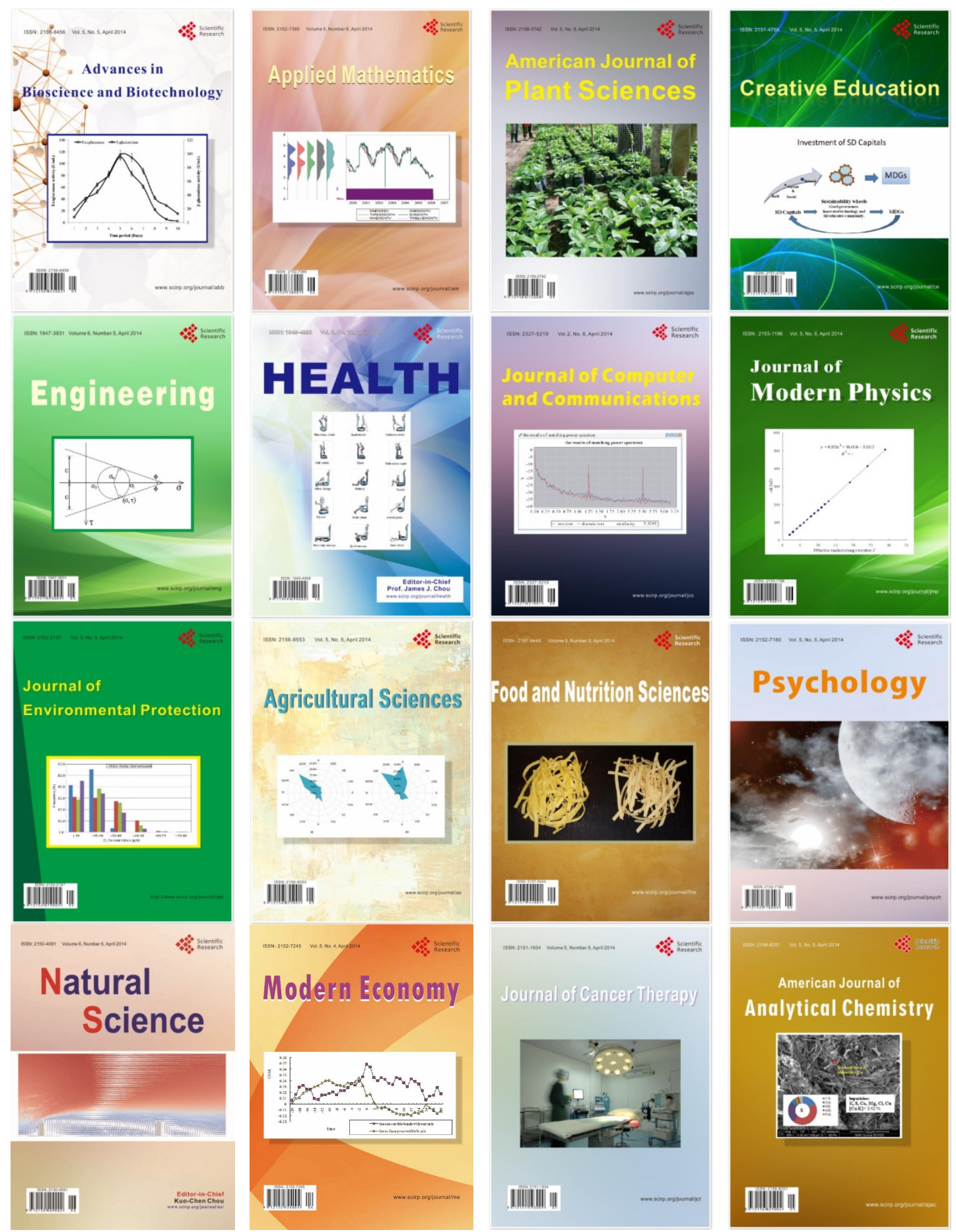